\title{
BMJ Open EvaLuation Using Cardiac Insertable Devices And TelephonE in Hypertrophic Cardiomyopathy (ELUCIDATE HCM) - rationale and design: a prospective observational study on incidence of arrhythmias in Sweden
}

\author{
Peter Magnusson,, ${ }^{1,2}$ Stellan Mörner ${ }^{3}$
}

To cite: Magnusson $\mathrm{P}$, Mörner S. EvaLuation Using Cardiac Insertable Devices And TelephonE in Hypertrophic Cardiomyopathy (ELUCIDATE HCM) —rationale and design: a prospective observational study on incidence of arrhythmias in Sweden. BMJ Open 2017;7:e019541. doi:10.1136/ bmjopen-2017-019541

- Prepublication history for this paper is available online. To view these files, please visit the journal online (http://dx.doi. org/10.1136/bmjopen-2017019541).

Received 8 September 2017 Revised 30 October 2017 Accepted 9 November 2017

CrossMark

${ }^{1}$ Cardiology Research Unit, Department of Medicine, Karolinska Institutet, Stockholm, Sweden

${ }^{2}$ Center for Research and Development, Uppsala University, Gävle, Sweden ${ }^{3}$ Department of Public Health and Clinical Medicine and Heart Center, Umeå University, Umeå, Sweden

Correspondence to Dr Peter Magnusson; peter.magnusson@ regiongavleborg.se

\begin{abstract}
Introduction Hypertrophic cardiomyopathy (HCM) is a heterogeneous disease associated with sudden cardiac death (SCD) mainly due to ventricular tachycardia (VT) or fibrillation even though life-threatening bradycardia occurs. Risk stratification takes several variables into consideration including non-sustained VT (NSVT). An implantable cardioverter defibrillator effectively prevents SCD. Atrial fibrillation (AF) is common among patients with HCM and warrants anticoagulation even without conventional risk factors according to European guidelines. Routinely, the evaluation of arrhythmias using a 48-hour ambulatory external monitor takes place every $6-24$ months if patients do not report palpitations. The remaining time the potential burden arrhythmia is unknown. Therefore, the aim of the present study is to assess NSVT and AF incidence during 18 months by an insertable cardiac monitor (ICM).
\end{abstract}

Methods Adult patients, aged 18-65 years, with a validated diagnosis of HCM are eligible for the study. The study sample is planned to include 30 patients. A Confirm Rx is implanted at the level of the fourth rib on the left side subcutaneously after local anaesthesia. The application for monitoring is installed in the patients' smartphone and symptoms registered by the patient activation and VT detection programmed as $160 \mathrm{bpm}$ during $\geq 8$ intervals. An AF episode is recorded based on $\geq 2$ min duration. Bradycardia is recorded at $\leq 40 \mathrm{bpm}$ or pause $\geq 3.0 \mathrm{~s}$. The patients are followed during 18 months before explant.

Ethics and dissemination The study was approved by The Regional Ethical Committee in Umeå (protocol number 2017/13-31). The study protocol, including variables and prespecified research questions, the study was registered at Clinical Trial Registration NCT03259113. Each patient is informed about the study in both oral and written form by a physician and included after written consent.

\section{INTRODUCTION}

The hypertrophic cardiomyopathy (HCM) phenotype in adults requires at least $15 \mathrm{~mm}$

\section{Strengths and limitations of this study}

- An insertable cardiac monitor (ICM) allows for monitoring of atrial fibrillation, significant bradycardia and non-sustained ventricular tachycardia during 18 months.

- The study will evaluate true incidence of arrhythmia including symptom registration using a smartphone.

- Non-sustained ventricular tachycardia is a marker of sudden cardiac death, but the decision to implant a defibrillator should be carefully evaluated using guidelines as an ICM is currently not an indication as a diagnostic tool in this regard.

- The costly and invasive ICM may need to be studied in larger hypertrophic cardiomyopathy cohorts to allow for subgroup analyses and to generalise findings.

thickness of the myocardial wall deemed unexplained by other myocardial diseases and abnormal loading conditions due to hypertension or aortic stenosis. ${ }^{1}$ The point prevalence is approximately $1: 500$ in the general population but more than double if genotypes are also included..$^{2-4}$ Patients present with unspecific symptoms such as dyspnoea, chest discomfort, palpitations and dizziness. Echocardiogram is the cornerstone in diagnosing HCM but integrated medical information and awareness of differential diagnoses are important to avoid misclassification. ${ }^{5-7}$ The severity and pattern of hypertrophy and disease progression varies considerably. ${ }^{5}$ Life expectancy in general HCM cohorts seems to be acceptable but end-stage heart failure, cardiac embolisation 
stroke and devastating arrhythmias remain a challenge to improve prognosis. ${ }^{8-12}$

Sudden cardiac death (SCD) can be prevented by an implantable cardioverter defibrillator (ICD). In a recent review of patients with HCM and ICDs, $4.8 \%$ experienced appropriate therapy annually due to ventricular tachycardia (VT) or fibrillation and antitachycardia pacing or cardioversion rarely fails in terminating the arrhythmia. ${ }^{13}$ The decision to implant an ICD as primary prevention of SCD is based on evaluation of risk markers according to guidelines. ${ }^{1415}$ In both 2011 and 2014 guidelines, non-sustained VT (NSVT) is part of risk stratification based on evidence from several studies. ${ }^{16-19}$ It is more common at an older age and correlates with increased left ventricular wall thickness. ${ }^{1620}$ NSVT may be revealed during an ambulatory ECG, telemetry in the ward or during exercise test. HCM guidelines advocate follow-up including 48 hours ambulatory ECG whenever onset of palpitations or otherwise every 12-24 months but 6-12 months if the patient is in sinus rhythm and has enlarged left atrial diameter which predisposes for atrial fibrillation (AF) and SCD. ${ }^{15}$ This implies that during the remaining time, the presence of NSVT/AF is unknown.

In HCM, AF is known to worsen symptoms due to vulnerability to increased heart rate and lack of atrial filling. Furthermore, AF associated with ischaemic stroke or systemic embolisation is a major cause of death in HCM, which warrants attention to detection methods with increased sensitivity. ${ }^{11}$ In fact, according to current HCM guidelines, a history of AF, even without any CHA2DS2-VASc risk factor is an indication of anticoagulation. $^{15}$

The insertable cardiac monitor (ICM) Confirm Rx (Abbott/St Jude Medical, St Paul, Minnesota, USA) provides long-term monitoring of atrial and ventricular arrhythmias in addition to bradycardia. ${ }^{21}$ This device could potentially reveal the true incidence of arrhythmia in patients with HCM which is the rationale of the present study: EvaLuation Using Cardiac Insertable Devices And TelephonE in Hypertrophic Cardiomyopathy (ELUCIDATE HCM).

\section{OBJECTIVES}

Primary objective is to assess the incidence of NSVT during 18 months follow-up using an ICM.

Secondary objective is to assesses the incidence of $\mathrm{AF}$ during 18 months follow-up using an ICM.

\section{METHODS}

\section{Setting and selection}

Adult patients with a confirmed diagnosis of HCM will be recruited from the catchment area of Region Gävleborg and Umeå University Hospital (tertiary centre) in northern Sweden. Eligible patients are identified from hospital databases (diagnostic codes I42.1 or I42.2) and validated using medical records. The recruitment started in August 2017. The device will be implanted in addition to standard care.

\section{Inclusion and exclusion}

Patients, aged 18-65 years, with a confirmed diagnosis of HCM are eligible for the study. Exclusion criteria as follows: aortic stenosis (moderate, severe), hypertrophy associated with metabolic disease (eg, Fabry-Andersen) and syndromes (eg, Noonan syndrome), systolic heart failure with ejection fraction $\leq 55 \%$, pacemaker, implantable defibrillator, myocardial infarction, percutaneous coronary intervention, coronary bypass grafting, pulmonary vein isolation, Maze surgery, VT ablation, ectopic atrial tachycardia ablation, renal clearance $\leq 40 \mathrm{~mL}$ / min (Cockcroft-Gault Equation), malignancy or other comorbidity with $\leq 5$ years life expectancy, pregnancy (or planned $\leq 18$ months), drug addition, severe psychiatric disease, not able to participate in 18 months follow-up, 5-year risk of sudden cardiac death $\geq 6 \%$ according to the risk calculator. ${ }^{19}$ Myectomy or alcohol septal ablation is not an exclusion criterion.

\section{Implantation and monitor setup}

The implantation procedure is performed in local infiltrative anaesthesia (carbocaine with epinephrine) using the standard operation kit for Confirm Rx via a $5 \mathrm{~mm}$ incision at the level of the fourth rib on the left side subcutaneously. The application for monitoring is installed on the patients' own smartphone or a one that will be lent during the study period. The connection to the home-monitoring site Merlin is administrated and the patient is instructed how to use application and report potential symptoms according to guidelines. ${ }^{22}$ Postprocedure, paracetamol (acetaminophen) is recommended to control pain.

\section{Programming}

VT detection is programmed as $160 \mathrm{bpm}$ during $\geq 8$ intervals with high electrogram (EGM) priority and discriminator sudden onset activated (onset delta 18\%) a bigemini qualifier off. An AF episode is recorded based on $\geq 2$ min duration (the shortest programmable duration), AF-burden $\geq 6$ hours a day or ventricular rate during AF $100 \mathrm{bpm}$ for 6 hours daily. Bradycardia is recorded at $\leq 40 \mathrm{bpm}$ or pause $\geq 3.0 \mathrm{~s}$. Patient-activated symptom episodes have high EGM priority with 6 min symptom pretrigger duration and $1 \mathrm{~min}$ symptom post-trigger duration and the first 8 EGMs are stored. Maximal ventricular sensitivity is typically $0.15 \mathrm{mV}$ but adjusted if R-waves are low. Threshold start is $75 \%$ and sense refractory period $250 \mathrm{~ms}$ and decay delay $60 \mathrm{~ms}$.

\section{Follow-up}

Patients are encouraged to report symptoms by using the smartphone application. In addition, every third month there is an automatic interrogation of the device and transfer to the home-monitoring site Merlin, which is reviewed every second day except for weekends. False detection of arrhythmia by the device is expected to be 
frequent based on experience. Therefore, all episodes are scrutinised as part of work process. At 18 months, the device is explanted. Patients are scheduled for follow-up every third months but detection of arrhythmia warrants contact with the patient as part of clinical management.

\section{Power analysis}

A power analysis is based on previous research findings and estimation of outcome with certain relevance of hypothesis testing. This is the first study on incidence of NSVT and AF in HCM using continuous monitoring for a period of 18 months. Thus, the diagnostic yield is unknown. A formal power analysis have not been conducted and estimation of sample size is based on clinical judgement and available resources.

\section{Statistics}

Numerical data will be presented as frequencies, percentages, means and percentiles. Continuous variables are summarised as means, SD and percentiles and compared using t-tests. The $\chi^{2}$ test is used for categorical variables. Kaplan-Meier estimates is used to describe time-to-event analysis (time from implant to AF and NSVT, respectively, and cumulative incidence at 6,12 and 18 months will be reported).

A two-sided $\mathrm{P}$ value of $<0.05$ is considered statistically significant. The database will be stored in Excel 2010 and imported into SPSS V.22 (IBM) for statistical analyses.

\section{Variables}

Patient characteristics at enrolment as follows: age, family history of SCD, unexplained syncope, known HCM associated mutation, weight, length, NSVT at 48 hours ECG. Echocardiography parameters such as maximal wall thickness, left ventricular outflow tract gradient and left atrial size will be recorded.

\section{Ethics and dissemination}

This study is conducted in accordance with the Declaration of Helsinki. ${ }^{23}$ The study protocol, including variables and prespecified research questions, the study was registered at Clinical Trial Registration NCT03259113 and approved on 24 August 2017. Documentation of research data and management of study follow the Guideline for Good Clinical Practice. ${ }^{24}$ Each patient is informed about the study in both oral and written form by a physician and included after written consent. After the follow-up period is completed, the database will be closed and followed by statistical work, interpretation of results and dissemination to a scientific journal.

\section{DISCUSSION}

This is the first study on arrhythmia detection in HCM using an ICM. These devices offer the superior advantage of prolonged monitoring in comparison to external loop recorders. ICM is currently used in certain cases of HCM such as syncope evaluation or possibly in patients with frequent palpitations but not in routine evaluation.
The application of ICM in the specific group of HCM has been proposed already in statements of research priorities. $^{25}$

The newly launched Confirm Rx uses a smartphone application that is used instead of a standard home monitor and provide the possibility of remote monitoring and symptom registration.

Until now, 48 hours ambulatory monitoring is used, but often telemetry in hospital wards detect arrhythmias in HCM. That means that these modes of monitoring may coincidentally detect AF and VT but with increased likelihood in patients with higher incidence of arrhythmias. It is unclear if a higher incidence of arrhythmia reflects a higher risk of SCD/stroke, and our study has a small sample size and no long-term follow-up of outcomes of SCD. Even though this study will elucidate the true arrhythmia incidence, the clinical interpretation will remain partly unsolved. In addition, ICMs are costly devices and health economy analyses will be needed before potential use in broader HCM groups.

Detection of AF is important in HCM because of the increased of embolic stroke. ${ }^{11}$ AF detection by ICM is superior compared with standard evaluation of monitoring in cryptogenic stroke. ${ }^{26}$ However, it should be remembered that clinical management of patients whose $\mathrm{AF}$ was detected at pacemaker interrogation is controversial, but there is compelling evidence when duration is at least 6 min. ${ }^{27}$ Patients with silent AF, that is, asymptomatic still carry a considerable risk and should be managed the conventional way with regard to anticoagulation. ${ }^{27}$ Even though the definition of $\mathrm{AF}$ is an atrial electrical activity of $\geq 300 \mathrm{bpm}$ during $\geq 30 \mathrm{~s}$, devices such as pacemaker register atrial high rate and the benefit of apixaban is currently studied. ${ }^{28}$ The correlation to symptoms is possible in our study and future smartphone-based ICM usage is promising. ${ }^{29}$

Safety aspects during the study imply referral to the patients' physician for anticoagulation if AF is detected, sustained VT/ventricular fibrillation for decision-making with regard to an ICD and if a significant bradycardia occurs that implies a pacemaker indication. If an ICD or pacemaker is indicated, the ICM will remain until study ends at 18 months.

Future research of larger series of patients with HCM using ICMs with a larger sample size allowing for analysis of subgroups like patients who have undergone myectomy or alcohol septal ablation are welcomed. Interestingly, the ICM size has become reduced over time, and implantation techniques have become simpler, which allows for more favourable cost-benefit as implantation outside the electrophysiological laboratory is possible.

Still, this is the first study on arrhythmia burden and symptoms in consecutive unselected patients with HCM using an ICM connected to a smartphone for monitoring and symptom elucidation.

Acknowledgements The authors acknowledge Anders Edström, Abbott who provided support and guidance. 
Contributors PM: idea, design, project management and writing the manuscript. SM: critical revision of the manuscript and project management.

Funding Region Gävleborg funded this research project and Abbott/St Jude Medical provided funding for the Confirm Rx (grant number 84540).

Competing interests None declared.

Ethics approval The study was approved by the Regional Ethical Committee in Umeå on 7 February 2017 (protocol number 2017/13-31).

Provenance and peer review Not commissioned; externally peer reviewed.

Open Access This is an Open Access article distributed in accordance with the Creative Commons Attribution Non Commercial (CC BY-NC 4.0) license, which permits others to distribute, remix, adapt, build upon this work non-commercially, and license their derivative works on different terms, provided the original work is properly cited and the use is non-commercial. See: http://creativecommons.org/ licenses/by-nc/4.0/

(c) Article author(s) (or their employer(s) unless otherwise stated in the text of the article) 2017. All rights reserved. No commercial use is permitted unless otherwise expressly granted.

\section{REFERENCES}

1. Elliott P, Andersson B, Arbustini E, et al. Classification of the cardiomyopathies: a position statement from the European Society of Cardiology Working Group on Myocardial and Pericardial Diseases. Eur Heart J 2008;29:270-6.

2. Maron BJ, Gardin JM, Flack JM, et al. Prevalence of hypertrophic cardiomyopathy in a general population of young adults. Echocardiographic analysis of 4111 subjects in the CARDIA Study. Coronary artery risk development in (young) adults. Circulation 1995;92:785-9.

3. Zou Y, Song L, Wang Z, et al. Prevalence of idiopathic hypertrophic cardiomyopathy in China: a population-based echocardiographic analysis of 8080 adults. Am J Med 2004;116:14-18.

4. Semsarian C, Ingles J, Maron MS, et al. New perspectives on the prevalence of hypertrophic cardiomyopathy. J Am Coll Cardiol 2015;65:1249-54.

5. Klues HG, Schiffers A, Maron BJ. Phenotypic spectrum and patterns of left ventricular hypertrophy in hypertrophic cardiomyopathy: morphologic observations and significance as assessed by twodimensional echocardiography in 600 patients. J Am Coll Cardiol 1995;26:1699-708.

6. Losi MA, Nistri S, Galderisi M, et al. Echocardiography in patients with hypertrophic cardiomyopathy: usefulness of old and new techniques in the diagnosis and pathophysiological assessment. Cardiovasc Ultrasound 2010;8:7.

7. Magnusson P, Palm A, Branden E, et al. Misclassification of hypertrophic cardiomyopathy: validation of diagnostic codes. Clin Epidemiol 2017;9:403-10.

8. Maron BJ, Rowin EJ, Casey SA, et al. Hypertrophic cardiomyopathy in adulthood associated with low cardiovascular mortality with contemporary management strategies. J Am Coll Cardiol 2015;65:1915-28.

9. Maron BJ, Olivotto I, Spirito P, et al. Epidemiology of hypertrophic cardiomyopathy-related death: revisited in a large non-referral-based patient population. Circulation 2000;102:858-64.

10. Pasqualucci D, Fornaro A, Castelli G, et al. Clinical spectrum, therapeutic options, and outcome of advanced heart failure in hypertrophic cardiomyopathy. Circ Heart Fail 2015;8:1014-21.
11. Guttmann OP, Rahman MS, O’Mahony C, et al. Atrial fibrillation and thromboembolism in patients with hypertrophic cardiomyopathy: systematic review. Heart 2014;100:465-72.

12. Magnusson P, Gadler F, Liv P, et al. Causes of death and mortality in hypertrophic cardiomyopathy patients with implantable defibrillators in Sweden. J Cardiovasc Med 2016;17:478-84.

13. Wang N, Xie A, Tjahjono R, et al. Implantable cardioverter defibrillator therapy in hypertrophic cardiomyopathy: an updated systematic review and meta-analysis of outcomes and complications. Ann Cardiothorac Surg 2017;6:298-306.

14. Gersh BJ, Maron BJ, Bonow RO, et al. ACCF/AHA guideline for the diagnosis and treatment of hypertrophic cardiomyopathy: executive summary: a report of the American College of Cardiology Foundation/American Heart Association Task Force on Practice Guidelines. Circulation 2011;2011:2761-96.

15. Elliott PM, Anastasakis A, Borger MA, et al. ESC Guidelines on diagnosis and management of hypertrophic cardiomyopathy: the task force for the diagnosis and management of hypertrophic cardiomyopathy of the European Society of Cardiology (ESC). Eur Heart J 2014;2014:2733-79.

16. Monserrat L, Elliott PM, Gimeno JR, et al. Non-sustained ventricular tachycardia in hypertrophic cardiomyopathy: an independent marker of sudden death risk in young patients. J Am Coll Cardiol 2003;42:873-9.

17. Adabag AS, Casey SA, Kuskowski MA, et al. Spectrum and prognostic significance of arrhythmias on ambulatory Holter electrocardiogram in hypertrophic cardiomyopathy. J Am Coll Cardiol 2005;45:697-704.

18. Schinkel AF, Vriesendorp PA, Sijbrands EJ, et al. Outcome and complications after implantable cardioverter defibrillator therapy in hypertrophic cardiomyopathy: systematic review and meta-analysis. Circ Heart Fail 2012;5:552-9.

19. O'Mahony C, Jichi F, Pavlou M, et al. A novel clinical risk prediction model for sudden cardiac death in hypertrophic cardiomyopathy (HCM risk-SCD). Eur Heart J 2014;35:2010-20.

20. O'Hanlon R, Grasso A, Roughton M, et al. Prognostic significance of myocardial fibrosis in hypertrophic cardiomyopathy. J Am Coll Cardiol 2010;56:867-74.

21. Nölker G, Mayer J, Boldt LH, et al. Performance of an implantable cardiac monitor to detect atrial fibrillation: results of the DETECT AF Study. J Cardiovasc Electrophysiol 2016;27:1403-10.

22. Dubner S, Auricchio A, Steinberg J, et al. ISHNE/EHRA expert consensus on remote monitoring of cardiovascular implantable electronic devices (CIEDs). Europace 2012;2012:278-93.

23. World Medical Association. Declaration of Helsinki: ethical principles for medical research involving human subjects. J Postgrad Med 2002;2002:206-8.

24. European Medicines Agency. ICH harmonised tripartite guideline E6. In: European Medicines Agency, eds. Note for guidance on good clinical practice (PMP/ICH/135/95), 2002.

25. Force T, Bonow RO, Houser SR, et al. Research priorities in hypertrophic cardiomyopathy: report of a Working Group of the National Heart, Lung, and Blood Institute. Circulation 2010;122:1130-3.

26. Sanna T, Diener HC, Passman RS, et al. Cryptogenic stroke and underlying atrial fibrillation. N Engl J Med 2014;370:2478-86.

27. Healey JS, Connolly SJ, Gold MR, et al. Subclinical atrial fibrillation and the risk of stroke. N Engl J Med 2012;366:120-9.

28. U.S National Library of Medicine. Apixaban for the Reduction of Thrombo-Embolism in Patients with Device-Detected Sub-Clinical Atrial Fibrillation (ARTESiA). https://clinicaltrials.gov/ct2/show/ NCT01938248 (accessed 2 Oct 2017).

29. Mittal S. Smartphone-based electrocardiographic and cardiac implantable electronic device monitoring. Cardiol Rev 2017;25:12-16. 